Utah State University

DigitalCommons@USU

Space Dynamics Lab Publications

Space Dynamics Lab

$1-1-2011$

\title{
Testing of Highly Accurate Blackbodies
}

\author{
Harri Latvokoski \\ Utah State University \\ Mike Watson \\ Utah State University \\ Shane Topham \\ Utah State University
}

Follow this and additional works at: https://digitalcommons.usu.edu/sdl_pubs

\section{Recommended Citation}

Latvokoski, Harri; Watson, Mike; and Topham, Shane, "Testing of Highly Accurate Blackbodies" (2011).

Space Dynamics Lab Publications. Paper 77.

https://digitalcommons.usu.edu/sdl_pubs/77

This Article is brought to you for free and open access by the Space Dynamics Lab at DigitalCommons@USU. It has been accepted for inclusion in Space Dynamics Lab Publications by an authorized administrator of DigitalCommons@USU. For more information, please contact digitalcommons@usu.edu.

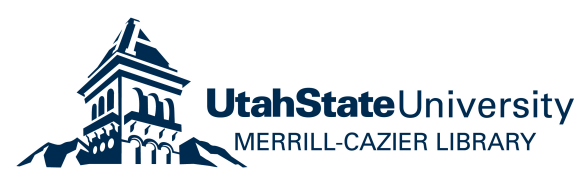




\title{
Testing of Highly Accurate Blackbodies
}

\author{
Harri Latvakoski, Mike Watson, and Shane Topham \\ Space Dynamics Laboratory / Utah State University \\ 1695 Research Park Way, N. Logan, UT 84341
}

\begin{abstract}
Many organizations, including Space Dynamics Laboratory, have built blackbodies with calculated emissivities of 0.995 to 0.9999 and estimated radiance temperature uncertainties of a few hundred $\mathrm{mK}$ or less. However, the calculated performance has generally not been demonstrated through testing or comparison with other highperformance blackbodies. Intercomparison is valuable; historically, when equipment or experimental results have been intercompared they are often found to disagree by more than the claimed uncertainties. Blackbody testing has been limited because testing at the required accuracy ( $0.1 \%$ or better in radiance) is a significant expense. Such testing becomes essential when proven, SI-traceble, absolute accuracy is required, such as for the CLARREO mission, which has an absolute accuracy requirement of $0.1 \mathrm{~K}$ (3 sigma) at $220 \mathrm{~K}$ over most of the thermal infrared and needs high-performance blackbodies to support this requirement. Properly testing blackbodies requires direct measurement of emissivity and accurate measurement of radiance or comparison of radiance from two blackbodies. This presentation will discuss these testing needs, various types of testing, and test results for a CLARREO prototype blacbkody.
\end{abstract}

Keywords: Blackbody, emissivity, SI-traceable, radiance uncertainty, temperature uncertainty, CLARREO

\section{INTRODUCTION}

Highly accurate blackbodies are needed to meet the strict accuracy requirements of modern radiometer and spectrometer instruments. For example, the Climate Absolute Radiance and Refractivity Observatory (CLARREO) ${ }^{1}$ mission is to measure the Earth's emitted spectral radiance from orbit for 5 years with an absolute accuracy of $0.1 \mathrm{~K}(3 \sigma)$ at $\sim 180$ to $\sim 320 \mathrm{~K}$ over most of the thermal infrared. To meet this requirement, CLARREO needs an on-board blackbody and ground calibrator blackbody with accuracy greater than this. While CLARREO has the strictest accuracy requirements of any mission currently planned, other Earth observing mission have accuracy requirements approaching those of CLARREO.

Space Dynamics Lab (SDL) of Utah State University has considerable experience in building highly accurate blackbodies and has recently built a prototype blackbody to show the ability to support the CLARREO accuracy requirements. This blackbody was developed as part of the NASA Langley IIP program, Calibrated Observations of Radiance Spectra from the Atmosphere in the far Infrared (CORSAIR), and is referred to as the CORSAIR blackbody. ${ }^{2}$ Based on modeling, we expect this blackbody to have an emissivity of $\geq 0.9999$ from 5 to $50 \mu \mathrm{m}$ and errors from temperature sensors and gradients $<30 \mathrm{mK}$.

In general, blackbody performance is calculated from physical models and these results are then quoted as the blackbody performance. The blackbody performance is typically not tested nor compared with other blackbodies because of the significant expense involved, especially at the levels required for highly accurate blackbodies. Intercomparison is valuable; historically, when equipment or experimental results have been intercompared they are often found to disagree by more than the claimed uncertainties. When SI traceability is needed, proper blackbody testing is required. SI traceability is of benefit to any Earth observing missions attempting to contribute to a long record of observations, such as CLARREO.

\section{BLACKBODY TESTING}

Blackbody testing is often taken to mean end-to-end performance testing. The obvious complete performance test is observation of two blackbodies in succession with an accurate radiometer. This test directly compares the overall 
performance of one blackbody to another, and can be used to compare a new design to a known, trusted standard, or to compare two designs both believed to be highly accurate. Note that this comparison can not prove that a new design claiming higher accuracy than previous blackbodies meets its requirements, but it will show whether or not it is consistent with existing standards. To perform this test requires a highly accurate radiometer to make the comparison and a setup that provides a low, stable background environment (generally cryogenic) during the test. An alternative end-to-end test is to measure the output of the blackbody with an absolutely calibrated radiometer based on an electrical substitution radiometer. This test also requires an accurate radiometer and a low stable background during the test.

In addition to the complete end-to-end testing, various tests of individual blackbody properties are also possible. These tests range from complicated to relatively straightforward but are generally simpler to perform than complete performance tests. The simplicity of the test in some cases depends on the design, so a blackbody can be designed for ease of testing some properties. This was considered for the design of the CORSAIR blackbody since SI-traceability is important for CLARREO.

The sources of uncertainty in blackbody performance can be grouped into three categories: emissivity knowledge, errors in the temperature sensor readings, and temperature gradients between the sensors and critical surfaces. Each of these can be tested at some level to show if performance goals are met. While test of individual characteristics can not prove the overall performance of the blackbody, they can increase confidence in the calculated values.

\section{THE CORSAIR BLACKBODY}

The CORSAIR blackbody (shown in Figure 1) has been described extensively elsewhere. ${ }^{2}$ The blackbody achieves high emissivity by using specular paint in a re-entrant cone cavity design. The paint has a low diffuse reflectivity, which directly reduces the blackbody reflectivity, and the cavity design forces any ray entering the blackbody at near normal incidence to reflect more than five times before exit, thereby keeping any blackbody reflectance due to paint specular reflectance insignificant. Various design features keep gradients between temperature sensors and the viewed surfaces inside the blackbody very low, such as the thick cone, several concentric cylinders around the inner cylinders, heat sinking of temperature sensor leads, and heaters kept away from the critical surfaces. The sensor mounting is designed to add no stress to the sensors when they are inserted since stress can affect the sensor readings. The CORSAIR blackbody operating specifications are given in Table 1.

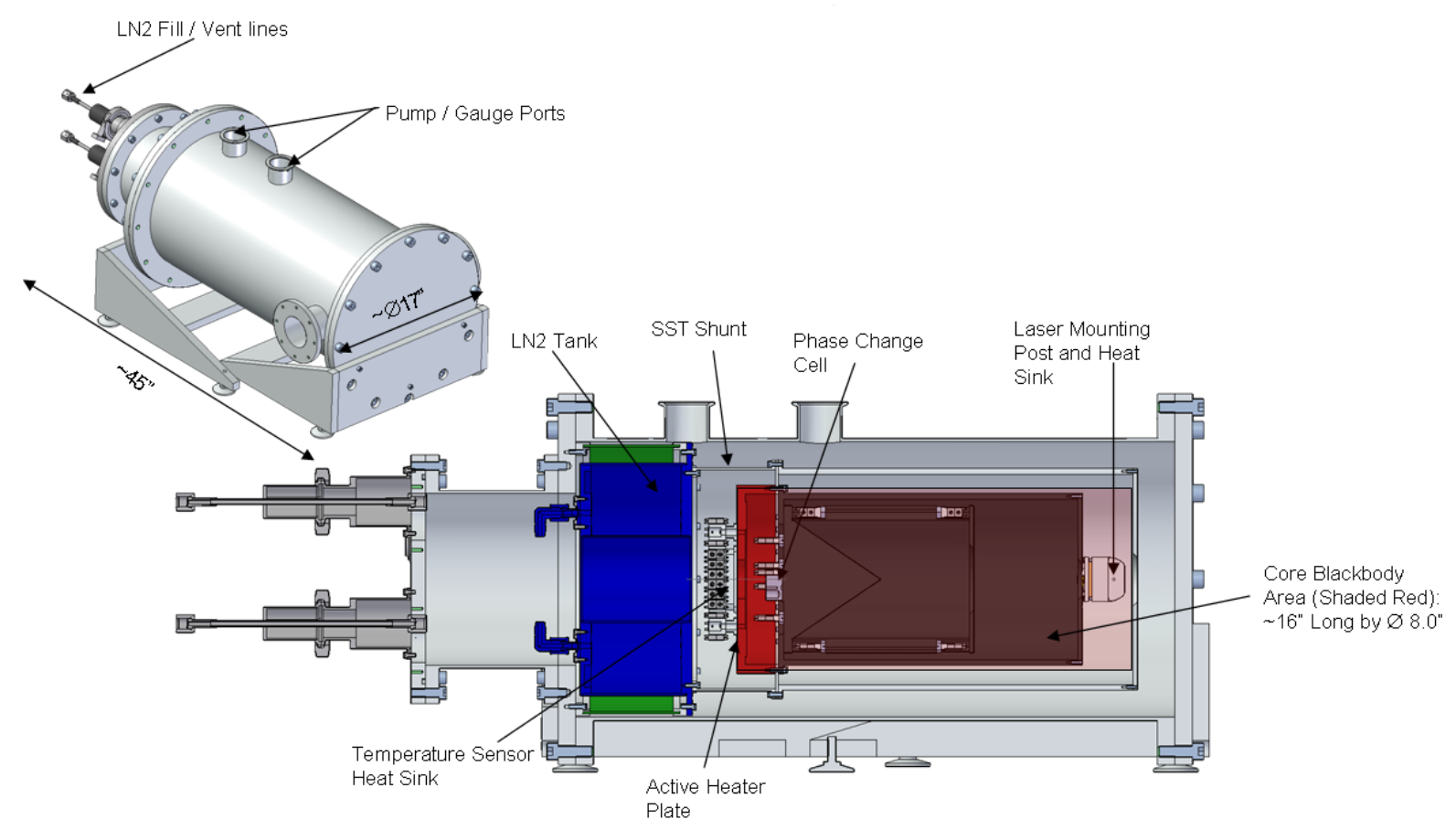

Figure 1 . The CORSAIR blackbody mechanical design. 
Table 1. CORSAIR blackbody operating specifications.

\begin{tabular}{|l|l|}
\hline Controlled temperature range & 200 to $350 \mathrm{~K}$ \\
\hline Operating Environment & Ambient or inside a larger vacuum chamber \\
\hline Coolant & Liquid Nitrogen \\
\hline Environment temperature & 77 K to ambient \\
\hline Aperture & 1.5 inches \\
\hline Beam divergence accommodated & $6^{\circ}$ full angle \\
\hline Emissivity & 0.9999 \\
\hline Temperature Uncertainties & $30 \mathrm{mK}$ \\
\hline
\end{tabular}

\section{BLACKBODY TESTING EXAMPLES}

This section describes several types of blackbody performance tests SDL has performed or collaborated on for the CORSAIR blackbody and other blackbodies. The data have not been completely processed for most tests so only preliminary results are presented.

\subsection{Temperature gradients}

Temperature gradients in the blackbody can be best tested by having several accurate temperature sensors in the blackbody at several locations where gradients may be a concern. The ability to detect gradients will be limited by the temperature sensor accuracy and by where sensors can be effectively placed. Not all gradients can be tested this way, such as those through the blackbody paint, but if a blackbody contains accurate sensors, this test is automatically performed whenever the blackbody is operated.

Figure 2 shows temperature sensor readings from the CORSAIR blackbody from two sensors in the blackbody cone and two at either end of the adjacent inner cylinder (Figure 1) when the blackbody is in thermal equilibrium. When viewing the blackbody, $\sim 89 \%$ of the observed light is emitted by the cone, with nearly all of the remaining $~ 11 \%$ emitted by the adjacent cylinder, thus, temperature gradients on the cone and cylinder would affect performance. The data in Figure 2 and similar data at other temperatures from -40 to $+40{ }^{\circ} \mathrm{C}$ shows that gradients are essentially non-existent in this blackbody.

\subsection{Temperature sensors}

Small industrial temperature sensors can be calibrated to high accuracy (better than $10 \mathrm{mK}$ ); however, when placed in a blackbody, the readings can be affected by drift in the sensors or mechanical stresses from mounting or handling. Using multiple, redundant temperature sensors in a blackbody can help determine if sensors have maintained their calibration. If all sensors agree when the blackbody is in equilibrium, as shown for the CORSAIR blackbody in Figure 2, it is likely they have maintained their calibration.

A better method for testing and maintaining temperature sensor calibration is to include phase change materials in a blackbody. These can be used to compare temperature sensor readings to the known melt point of the material. The CORSAIR blackbody includes a phase change cell ${ }^{3}$ that is temperature controlled independent of the blackbody, and contains pure $\mathrm{Hg}, \mathrm{H}_{2} \mathrm{O}$ and $\mathrm{Ga}$ in separate compartments. Since expansion of each material is monitored, the cell can be brought unambiguously to the melt point of each material, thereby providing absolute calibration for a temperature sensor in the cell. When the cell is returned to equilibrium with the blackbody, the calibration can be transferred to the blackbody temperature sensors. Because the cell provides an absolute reference independent of the temperature sensor calibration, it allows a consistency check of both the cell operation and the temperature sensors. Preliminary results of using the phase change cells in the CORSAIR blackbody show the temperature sensors are consistent with phase change cell results to within $\sim 20 \mathrm{mK}$ at the melt points of the materials. Prior to placement in the blackbody, the temperature sensors were absolutely calibrated to $\sim 15 \mathrm{mK}(\mathrm{k}=1)$. 


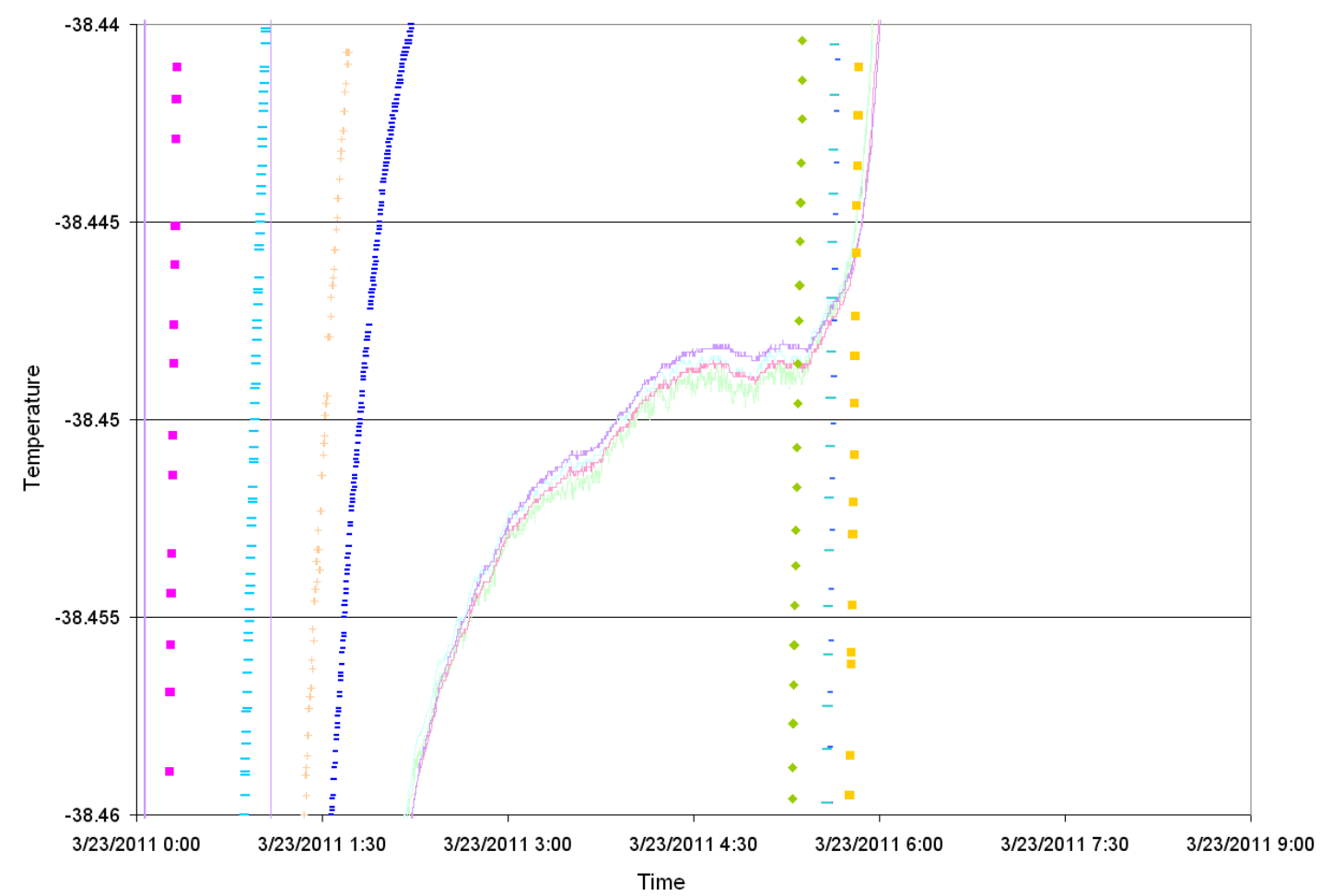

Figure 2. Temperature sensor readings from the CORSAIR blackbody cone and cylinder (the 4 curves in the middle) when operated at $-38.5 \mathrm{C}$. The sensors agree to within $\sim 1 \mathrm{mK}$, which is the limit of the sensor intercalibration.

\subsection{Emissivity testing}

For a highly accurate blackbody, the emissivity must either be very high or at least well known. There are several methods to measure emissivity. A common method is to observe the blackbody with an instrument through a collar around the entrance aperture, where the collar temperature can be controlled. The collar temperature is raised and the resulting change in observed signal is measured and related to the blackbody reflectivity. This method is known as a heated halo test and has been used effectively for emissivity measurement at SDL and elsewhere.

For the CORSAIR blackbody, a method to monitor emissivity was required, but a heated halo was unlikely to provide enough power under CLARREO-like conditions to measure reflectivity at the required low level. Instead we included an alternative method to monitor emissivity based on a single quantum cascade laser (QCL) to test the concept ${ }^{2,4}$. The design of the monitor is simple; the QCL, with no optics, shines into the blackbody through a small hole near the aperture of the blackbody, and the instrument observing the blackbody is used as a detector. This monitor design also allows for a measure of the blackbody emissivity, since the laser with its broad beam mimics a uniform source entering the blackbody through its aperture. The emissivity monitor was used during the test of the CORSAIR blackbody with the SDL transfer radiometer described further in section 4.4. The CORSAIR blackbody was observed with a spectrometer and the laser was then turned on while spectra continued to be collected. The spectrometer was unable to detect a signal from the $9.56 \mu \mathrm{m}$ laser above its noise level. A preliminary calculation shows this is consistent with a blackbody emissivity of $\geq 0.999992$. This result is quite high, but is consistent with the model calculations and blackbody paint properties at this wavelength.

Emissivity can also be measured by the Complete Hemispherical Infrared Laser-based Reflectometer (CHILR) facility at NIST. CHILR works by shining a laser into the blackbody and using an integrating sphere to catch all the returned light, as shown in Figure 3. The result is compared to that obtained from a sample of known reflectance and used to calculate the blackbody emissivity. For CHILR, the measurement geometry is the reverse of how an instrument views the blackbody, but this is acceptable because of time invariance of light. The blackbody under test can be scanned and tilted 
to cover the range of view angles for the instrument to be used with the blackbody and the results averaged. For blackbodies with apertures smaller than the 2" aperture of the CHILR instrument, CHILR provides a direct measurement, while for larger blackbodies, calculations must be made to account for the missed light. CHILR has been used effectively to measure blackbody reflectivity to the $<10^{-4}$ level. For example, the on-board blackbody for the Cross-track Infrared Sounder (CrIS) instrument ${ }^{5}$ was recently measured with CHILR and preliminary results show it meets its required 0.995 emissivity and most likely its 0.0015 emissivity knowledge goals. ${ }^{6}$

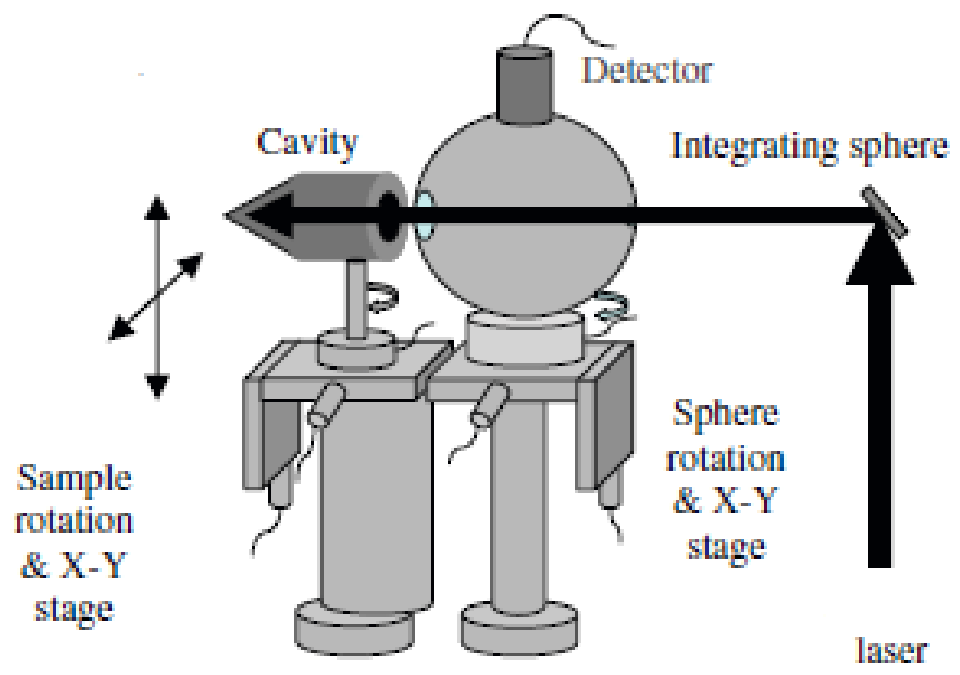

Figure 3. Diagram of the CHILR instrument to measure emissivity. The emissivity is found by comparing the observed return to that from a sample of known emissivity and an open hole measurement.

\subsection{Blackbody intercomparison}

We have recently preformed a comparison of the CORSAIR blackbody to our existing Long-wave Infrared Calibration Source (LWIRCS) blackbody using the SDL Transfer Radiometer (SXR). ${ }^{7}$ The SXR is a cryogenic radiometer and spectrometer combination housed inside a single vacuum chamber. In radiometer mode, light from the instrument under test passes through a set of filters on filter wheels to a single element Si:As BIB detector, which is cooled by liquid helium to $11 \mathrm{~K}$. There are 4 filter wheels with 8 slots each, allowing for 28 individual filters plus combinations in the 2 to $30 \mu \mathrm{m}$ range. Radiometer data is collected either in DC mode, or by using a tuning fork chopper in the beam and a lock-in amplifier to reduce noise. In spectrometer mode, the light is directed through a Fourier transform interferometer before reaching the filter wheel. The SXR contains a shutter and several stim source to track instrument offset and gain changes. All SXR options inside the chamber (choice of filter, radiometer or spectrometer mode, etc) are software selectable while the chamber is cold and under vacuum. Table 2 lists the SXR optical specification and Figure 4 is a picture of the CORSAIR blackbody attached to the SXR.

Table 2: SXR basic specifications operating specifications.

\begin{tabular}{|l|l|}
\hline Coolant & Liquid helium (11 K detector, 20 K bench) \\
\hline Aperture & $70 \mathrm{~mm}(2.76 ”)$ \\
\hline Beam divergence accommodated & $1 \mathrm{mrad}$ \\
\hline Wavelength range & 2 to $30 \mu \mathrm{m}$ \\
\hline Window (removable) & $\mathrm{KBr}$, heated to $310 \mathrm{~K}$ \\
\hline Spectrometer resolution & $0.5,1,2$, or $4 \mathrm{~cm}^{-1}$ \\
\hline Spectrometer sampling & Once per HeNe laser fringe \\
\hline
\end{tabular}




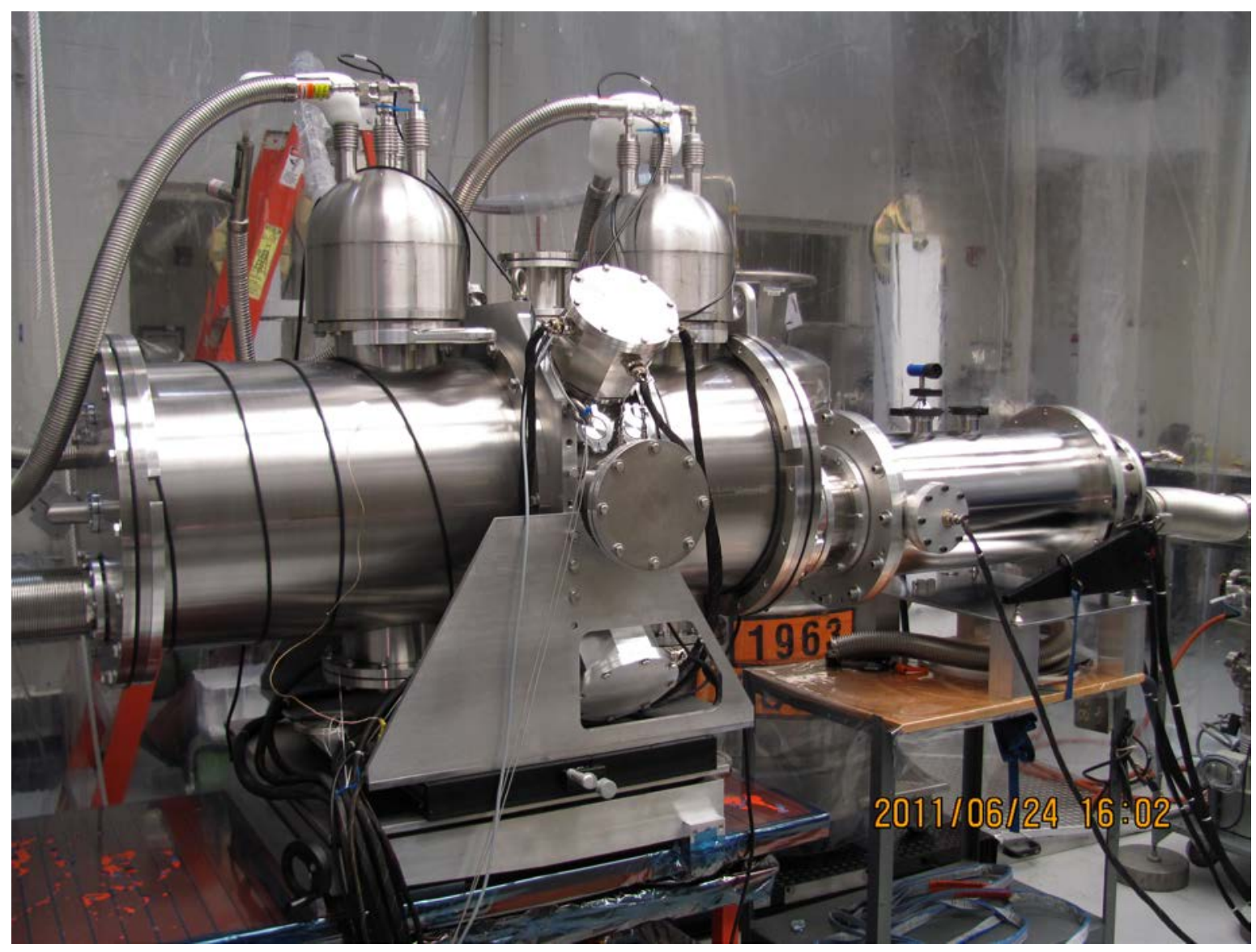

Figure 4. The CORSAIR blackbody (right) attached to the SXR (left).

The SXR aperture of 2.76" is larger than the normal 1.5" aperture of the CORSAIR blackbody, so for this test we changed the CORSAIR aperture to 3" by replacing two aperture plates. During this test the $\mathrm{KBr}$ window at the entrance of the SXR was left in place to avoid contamination of the SXR. For best performance, this window should be removed, and the presence of the window likely limited the test performance here. The SXR was first used to observe LWIRCS at 8 temperature set points through several filters in radiometer and spectrometer mode. After this was completed, LWIRCS was removed, and the CORSAIR blackbody was switched in without warming up the SXR. It was observed at 5 temperatures (a subset of the 8 used for LWIRCS) between 230 and $310 \mathrm{~K}$ through the same set of filters.

Quick-look analyses of narrow 8.4 and $12 \mu \mathrm{m}$ radiometer band observations show that emission from the LWIRCS and CORSAIR blackbodies, when near the same temperature, are consistent to under $1 \%$ at all temperature from 230 to 310 $\mathrm{K}$. The uncertainty for this quick-look is limited by round-off error in the written numbers.

\section{CONCLUSIONS}

Testing of highly-accurate blackbodies ranges from simple to complex and expensive but is certainly worthwhile to improve confidence in the blackbody performance. SDL has recently performed several types of blackbody testing, and future work will include proper processing of test results. We expect the test results will be useful, but the test accuracy may not reach the high levels required for CLARREO blackbodies. In this case, we will consider how to improve testing accuracies. 


\section{REFERENCES}

[1] http://clarreo.larc.nasa.gov/index.php

[2] Latvakoski, H., Watson, M., Topham, T.S., Scott, D., Wojcik, M., Bingham, G., "A high-accuracy blackbody for CLARREO," Proceedings of SPIE 7808, 78080X (2010).

[3] Topham, T.S., Bingham, G., Latvakoski, H., Watson, M., Ahlstrom, D., "Phase Transitions for On-orbit Temperature Recalibration," CALCON 2010.

[4] Lwin, M., Wojcik, M., Latvakoski, H., Scott, D., Watson, M., Marchant, A., Topham, S., Mlynczak, M., "Characterization of a quantum cascade laser-based emissivity monitor for CORSAIR," Proceedings of SPIE 8012, 80123M (2011).

[5] http://jointmission.gsfc.nasa.gov/cris.html

[6] Latvakoski, H., Hansen, L., Zeng, J. Scott, D., Bingham, G., "CHIRL measurement of the CrIS ICT," CALCON 2010.

[7] Johnson, K. B., Thurgood, A., Blakely, J., Tansock, J., “Space Dynamics Transfer Radiometer," CALCON 2009. 